\title{
The tune of sensory inputs
}

Many neurons in the sensory cortex are tuned to specific stimulus features, but the mechanisms underlying this phenomenon have been a topic of some debate. Now, Jia et al. have used a novel in vivo imaging technique to measure input signals in the visual cortex and have suggested a new model for signal integration at the level of the dendritic tree.

A classical example of sensory neuron tuning is the preferential firing of a subset of neurons in the visual cortex in response to a moving visual stimulus of a particular orientation. A popular model for the underlying mechanism has been the clustering of orientationspecific synaptic inputs on a given dendrite. To investigate these processes in a physiologically relevant

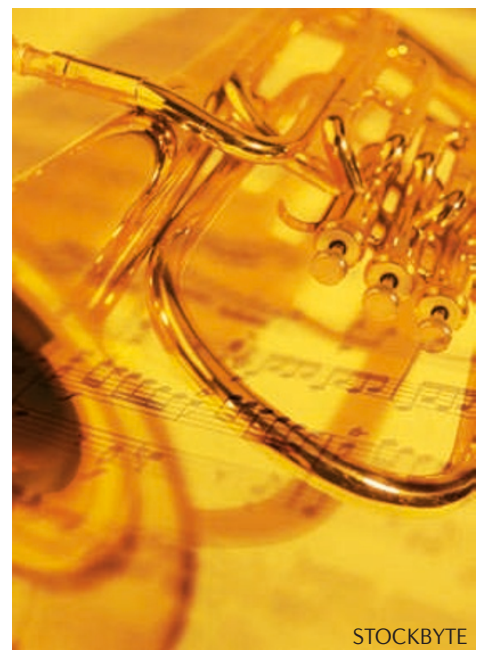

setting, the authors performed electrophysiological recordings in layer 2-3 neurons of the primary visual cortex in mice. Consistent with previous studies, the output firing of some visual cortex neurons was highly tuned to the orientation of a visual stimulus in the form of a drifting grating. To determine whether the sensory afferent signal in these neurons showed a similar orientation preference, the authors hyperpolarized the cells to $-70 \mathrm{mV}$ so that stimulation would not cause firing. In contrast to the output response, the input signal of subthreshold membrane depolarization was evoked by stimuli of various orientations.

Combination of a $\mathrm{Ca}^{2+}$-sensitive dye and two-photon microscopy revealed that the subthreshold depolarizations were associated with local $\mathrm{Ca}^{2+}$ transients, or 'hot spots', in neighbouring regions of the same dendrite. These transients were attenuated by the NMDA ( $N$-methyl$\mathrm{D}$-aspartate) receptor antagonist AP5, which suggests that they arose through glutamate-dependent synaptic transmission.

Next, the authors visualized the response to a drifting grating of various orientations in several dendrites of a given hyperpolarized neuron on multiple focal planes. In contrast to the whole-cell sensory afferent signal, hot spots showed a highly tuned preference for a specific stimulus orientation. Surprisingly, they seemed to be randomly distributed throughout the dendritic tree, such that local $\mathrm{Ca}^{2+}$ transients could be generated at various sites along a given dendrite in response to stimuli of different orientations. Furthermore, hot spots with a preference for a given orientation were located on various dendrites of a single neuron rather than being clustered on the same dendrite. This implies that the 'computational unit' in these neurons is the whole dendritic tree, rather than the individual dendrite. Interestingly, dendritic hot spots were found even in neurons that did not show a preference for stimulus orientation in terms of output firing.

Together, these findings suggest that neurons in the visual cortex receive preferentially tuned inputs throughout the dendritic tree, which are then integrated and processed to give the characteristic output firing in response to specific stimulus characteristics. The techniques used enable these processes to be studied in an in vivo setting with high spatiotemporal resolution, which could provide a valuable approach to identify the missing links between sensory afferent signals and output firing.

Katie Kingwell

ORIGINAL RESEARCH PAPER Jia, H. et al Dendritic organization of sensory input to cortical neurons in vivo. Nature 464, 1307-1312 (2010)

FURTHER READING Silver, R. A. Neuronal arithmetic. Nature Rev. Neurosci. 9 Jun 2010 (doi:10.1038/nrn2864) 